\title{
CALCULATION OF THE MAIN PARAMETERS OF A MAGNETIC FLUX TUBE WITHIN THE MODEL OF CYLINDRICALLY- SYMMETRIC EQUILIBRIA OF TWO-COMPONENT PLASMA
}

\author{
Turakulov Z.Y., Karachik N.V., Minenko E.P.
}

Ulugh Bek Astronomy Institute (UBAI), Tashkent, Uzbekistan

\section{РАСЧЕТ ОСНОВНЫХ ПАРАМЕТРОВ МАГНИТНОЙ ТРУБКИ В РАМКАХ МОДЕЛИ ЦИЛИНДРИЧЕСКИ-СИММЕТРИЧНОГО РАВНОВЕСНОГО СОСТОЯНИЯ ДВУХКОМПОНЕНТНОЙ ПЛАЗМЫ}

\author{
Туракулов 3.Я., Карачик Н.В., Миненко Е.П. \\ Астрономический институт АН РУз, Ташкент, Узбекистан
}

В данной работе представлена аналитическая модель магнитной трубки (MT), которая описывается как ичилндрически-симметричное равновесное состояния двухкомпонентной плазмы. Плазменный поток представляется в виде суперпозиции двух заряженных сжимаемых компонент плазмы, которые взаимодействуют только в условиях электромагнитного поля. Суммарная плотность электрического заряда принимается равной нулю. Компоненты плазмы - это смесь протонного и электронного газов, которые вращаясь вокруг общей оси с разными угловыми скоростями, производят ненулевую плотность тока обоих компонент, которая служит в качестве источника магнитного поля.

Граница МТ обусловлена расстоянием, на котором угловые скорости обнуляются. Равновесное состояние системы обеспечено вращением плазмы в системе трех сил - магнитной, центробежной и градиента давления. В нашем случае модель весьма идеализирована и ограничена условием того, что окружающая плазма однородна и неподвиюна. Несмотря на это, представленная модель, при заданных параметрах - даёт возможность расчёта основных параметров МТ.

\section{DOI: 10.31725/0552-5829-2019-411-414}

In this work, we propose one from possible options of build of model, that allows an almost analytical solution. In order to build an adequate model, it is practical to use the Maxwell equations in the MHD approximation.

A two-component plasma can be visioned as superposition of two compressible fluids carrying electric charge densities, which move independently and interact only via their common electromagnetic field. Simplest equilibria of such a matter with non-zero magnetic field possess cylindrical symmetry, hence, describe magnetic flux tubes. These descriptions make it possible to estimate main characteristics of tubes and link them to that of surrounding plasma. This way total magnetic energy per unit of length can be linked to pressure and density beyond the tube as necessary condition of equilibrium. Therefore, construction of equilibria of this sort is a possible approach to modeling solar magnetic flux tubes. In simplest case both plasma components rotate about common axis. 
As both of them carry electric charge, their rotation creates some current densities that produces magnetic field acts. Its strength is collinear to the axis. Thus, each component stays in equilibrium determined by balance of centrifugal, magnetic and pressure forces. The total charge density is assumed to be zero everywhere, so that possible electrostatic force is not taken into account.

The problem of equilibrium of the system under consideration is onedimensional in round cylinder coordinates $\{z, \rho, \varphi\}$ in which all functions to be found depend only on $\rho$. These functions characterize plasma components, their rotation and acting forces. Since the total charge density is zero everywhere and, as usual particles of components have charges $\pm e$ where $e$ stands for that of electron, total numbers of particles of the components in each spatial domain are equal. Therefore there exists a specific (numerical) density of both components $v(\rho)$. Mass densities of the components are given by this density as $m_{ \pm} v(\rho)$ where $m_{ \pm}$are masses of the particles. Temperatures of the components are assumed to be equal and same everywhere. Therefore partial pressures of the components are as in an ideal gas

$$
p_{ \pm}(\rho)=\tau v(\rho) .
$$

The components rotate with angular velocities $\Omega_{-} \pm(\rho)$ which also are functions to be found. Current densities of the components are

$$
J_{ \pm}(\rho)= \pm \operatorname{ev}(\rho) \rho \Omega_{ \pm}(\rho)
$$

and so that the total current density is

$$
J(\rho)=e v(\rho) \rho\left[\Omega_{+}(\rho)-\Omega_{-}(\rho)\right]
$$

hence, magnetic field strength $H(\rho)$ satisfies the equation

$$
\frac{d H}{d \rho}=\rho e v(\rho)\left[\Omega_{+}(\rho)-\Omega_{-}(\rho)\right] .
$$

(2.4) will be ones of Maxwell's equations.

Each of two components moves under action of three forces which are centrifugal force equal to $\rho \Omega^{2} m v$, magnetic force equal to $H J_{ \pm}$and gradient of pressure. Therefore conditions of their equilibrium are

$$
m_{ \pm} \Omega_{ \pm}^{2}(\rho) \rho v(\rho) \pm e \rho \mu_{0} H(\rho) v(\rho) \Omega_{ \pm}-\tau \frac{d v}{d \rho}=0 .
$$

Since $m_{-} \ll m_{+}$, M angular velocity of the electron component $m_{-} \Omega_{-}^{2}$ therefore equilibrium conditions for the electron component simplifies

$$
\tau \frac{d v}{d \rho}=-e \mu_{0} H(\rho) \Omega_{-}(\rho) \rho v(\rho) .
$$

If we express partial pressure from this, then equilibrium condition for the proton component takes the form 


$$
\Omega_{+}^{2}+\frac{e \mu_{0} H(\rho)}{m_{+}}\left(\Omega_{+}-\Omega_{-}\right)=0,
$$

and from here you can find the difference in angular velocities:

$$
\Omega_{+}-\Omega_{-}=-\frac{m_{+}}{e \mu_{0} H(\rho)} \Omega_{+}^{2} .
$$

In result, equation (4) takes the form

$$
\frac{d H}{d \rho}=\rho v \frac{m_{+} \Omega_{+}^{2}}{\mu_{0} H(\rho)},
$$

or finally

$$
\frac{1}{2} \frac{d H^{2}}{d \rho}=-\frac{\rho v m_{+}}{\mu_{0}} \Omega_{+}^{2} .
$$

It is convenient to introduce a new independent variable $x$ :

$$
x=\int_{0}^{\rho} d t t v(t)
$$

and rewrite the equations (5) и (7) in the form

$$
\begin{aligned}
& \tau \frac{d v}{d x}=-e \mu_{0} H(x) \Omega_{-}(x) \\
& \frac{d H}{d x}=e \Omega_{+}(x)
\end{aligned}
$$

And the equilibrium condition for the proton component should be replaced by the equation (6).

As a result, we have the following system of equations:

$$
\begin{aligned}
& \tau \frac{d v}{d x}=-e \mu_{0} H(x) \Omega_{-}(x) \\
& \frac{\mu_{0}}{2} \frac{d H^{2}}{d x}=-m_{+} \Omega_{+}^{2}(x) \\
& \Omega_{+}^{2}+\frac{e \mu_{0} H(x)}{m_{+}}\left(\Omega_{+}-\Omega_{-}\right)=0
\end{aligned}
$$

This system consists of three equations for four unknowns, therefore one of desired functions can be specified arbitrarily.

Suppose, the function $H(x)$ is specified explicitly. Then, explicit form of the function $\Omega_{+}(x)$ follows from the equation in the second line of the system (10). Now, as the function $\Omega_{-}(x)$ can be obtained from the equation in the third line:

$$
\Omega_{-}(x)=\Omega_{+}(x)\left(1+\frac{m_{+} \Omega_{+}(x)}{e \mu_{0} H(x)}\right) .
$$

And finally, it remains to obtain the function $v(x)$ by integrating in the first line 


$$
\nu(x)=-\frac{e}{\tau} \int_{0}^{x} d y H(y) \Omega_{-}(y) .
$$

The last step is to pass from the variable $x$ to the coordinate $\rho$ that can only be done numerically. We chose the function $H(x)$ as

$$
H=H_{0} \cos ^{2} \frac{l x \pi}{2}
$$

assuming that it has a maximum on the axis and decreases to zero at the magnetic flux tube boundary. We take the value of tension from observations of sunspots $H_{0}=\frac{2}{5} \cdot 10^{-8} \mathrm{~A} / \mathrm{m}$, temperature in the convection zone $T=10^{6} \mathrm{~K}$ and density $10 \mathrm{~kg} / \mathrm{m}^{3}$. In these conditions, the numerical density $v$ plasma is equal to $6 \cdot 10^{27} \mathrm{~m}^{-1}$ inside magnetic flux tube and $10^{27} \mathrm{M}^{-3}$ outside, also $\mathrm{l}^{-1} \approx 2 \cdot 10^{34} \mathrm{M}^{-1}$.

The content of the model constructed in this paper is completely predetermined by the analytical solution of the system of equations (8) and (10).

The system (10) it is completely solvable and gives all the desired values as a functions of the variable $x$, which be stay unknown. In the scope of the constructed semi-analytical model, the radius of the tube with the selected parameters turned out to be equal $2.06 \cdot 10^{6} \mathrm{M}$. The central part is characterized by an almost constant value of magnetic field strength and a plasma density close to zero. At the periphery, the plasma density rises sharply to an external value, while the magnetic field strength also drops sharply to zero. The model well explains the phenomenon buoyancy of magnetic tube, since it requires that the plasma density inside the tube be lower than outside it. As a result, the tube explodes as it moves upward, in this time the pressure around it decreases and reaches a certain critical value.

As a result, we have the model that determines the relationships between the characteristics of the tube and the surrounding plasma. For example, between the pressure and the maximum value of the magnetic force in the magnetic tube. Something parameters calculated within that analytical model will undoubtedly prove to be very instructive for the understanding of solar phenomena. 